\title{
Áreas propícias para o ataque de morcegos hematófagos Desmodus rotundus em bovinos na região de São João da Boa Vista, Estado de São Paulo ${ }^{1}$
}

\author{
Murilo N. Gomes ${ }^{2,3 *}$, Antonio M.V. Monteiro², Vladimir S. Nogueira Filho ${ }^{3}$ \\ e Celso A. Gonçalves ${ }^{3}$
}

\begin{abstract}
Gomes M.N., Monteiro A.M.V., Nogueira Filho V.S. \& Gonçalves C.A. 2007. [Areas prone for vampire bat (Desmodus rotundus) attack on cattle in the São João da Boa Vista region, State of São Paulo.] Áreas propícias para o ataque de morcegos hematófagos em bovinos na região de São João da Boa Vista, Estado de São Paulo. Pesquisa Veterinária Brasileira 27(7):307-313. Instituto Nacional de Pesquisas Espaciais, Cx. Postal 515, São José dos Campos, SP 12201-970, Brazil. E-mail: murilong@dpi.inpe.br

Supported by previous studies that selected areas prone for the development of Desmodus rotundus roosts and bovine rabies transmitted by vampire bats in São Paulo state, the Department of Agriculture of the State of São Paulo (CDA) has conducted interventions for bovine rabies control in areas mostly close to the main rivers of surveillance regions. Inquiries of the actual concepts led to the development of a descriptive model to analyze the vampire bat Desmodus rotundus attacks on cattle far beyond main rivers and water streams in the São João da Boa Vista region, São Paulo state. The model was elaborated using satellite images classification, parameters from previous studies about vampire bats behaviour, data from the CDA and the Brazilian Institute of Geography and Statistics (IBGE). All this information was used to build a geographical database and integrate in a Geographical Information System then analyzed through spatial statistical and analytical methods. The results showed that areas close to the main rivers of the towns Aguaí, Casa Branca, Santa Cruz das Palmeiras and Tambaú were found not to be under risk of bat attacks, suggesting that the hypothesis behind the CDA control areas definition does not hold when contracted with the obtained spatial data. The results indicate that the technicians from CDA need to reformulate their strategy for defining the surveillance of vampire bat populations and rabies control.
\end{abstract}

INDEX TERMS: Epidemiology, rabies, Desmodus rotundus, GIS, remote sensing.

RESUMO.- Baseada em estudos anteriores, a Coordenadoria de Defesa Agropecuária da Secretaria da Agricultura e Abastecimento do Estado de São Paulo (CDA) vinha realizando nas regiões administrativas onde é feita a vigilância da raiva em herbívoros o controle de populações de Desmodus rotundus em áreas próximas aos principais rios. A descoberta de abri-

\footnotetext{
${ }^{1}$ Recebido em 9 de novembro de 2006.

Aceito para publicação em 24 de abril 2007.

Trabalho financiado com recursos da FAPESP (Projeto 03-12319-0).

2 Instituto Nacional de Pesquisas Espaciais (INPE), Cx Postal 515, São José dos Campos, SP 12201-970. *Autor para correspondência: murilong@dpi.inpe.br

${ }^{3}$ Coordenadoria de Defesa Agropecuária da Secretaria de Agricultura e Abastecimento do Estado de São Paulo, Av. Brasil 3240, Campinas, SP 13073-001.
}

gos e de animais mordidos pelos morcegos vampiros em outras áreas, além das áreas estabelecidas de risco levaram a questionamentos dos métodos em vigência. Isso estimulou a elaboração de um modelo descritivo para analisar áreas de risco para o ataque espoliativo de morcegos hematófagos em bovinos na região de São João da Boa Vista, estado de São Paulo. Para tanto, foram utilizadas imagens de satélite, parâmetros de estudos anteriores sobre o comportamento dos morcegos vampiros, dados da CDA e da Fundação Instituto Brasileiro de Geografia e Estatística (IBGE). Todas as informações foram agrupadas em um banco de dados e integradas em um Sistema de Informação Geográfica. Contraditoriamente ao que se pensava, observou-se que áreas próximas aos principais rios da região das cidades de Aguaí, Casa Branca, Santa Cruz das Palmeiras e Tambaú não deveriam ser con- 
sideradas de risco para o ataque dos morcegos hematófagos em bovinos. Isso sugere que é necessária uma reavaliação dos métodos adotados para o controle das populações da espécie e enfermidade em questão.

TERMOS DE INDEXAÇÃO: Epidemiologia, raiva, Desmodus rotundus, SIG, imagem de satélite.

\section{INTRODUÇÃO}

A raiva é uma enfermidade infecciosa que afeta mamíferos e é causada por um vírus da família Rhabdoviridae, gênero Lyssavirus. A enfermidade caracteriza-se histologicamente pelo desenvolvimento de uma poliencefalomielite linfocitária e manifesta-se clinicamente por sinais neurológicos, incluindo estado de excitação e paralisias diversas (Brass 1994). Desde os tempos mais remotos, a raiva vem sendo associada à transmissão pelos cães (Brass 1994). A partir de 1911, Carini ao estudar uma epidemia entre bovinos em Santa Catarina verificou que a enfermidade poderia ser transmitida por morcegos da espécie Desmodus rotundus E. Geoffroy, 1810 (Almeida et al. 1994). Entre os morcegos, os hematófagos dessa espécie são considerados os principais agentes transmissores da raiva aos bovinos (Acha \& Malaga-Alba 1988), os quais se encontram distribuídos desde o sul do México até o norte da Argentina (Uieda 1987).

Estudos para o controle dessa enfermidade vêm sendo direcionados paralelamente aos de comportamento, controle e dinâmica de populações do morcego $D$. rotundus e, conseqüientemente, de aspectos geográficos que favorecem a existência de abrigos em certas áreas, em detrimento de outras. A espécie pode habitar abrigos artificiais (bueiros, casas e minas abandonadas) assim como, abrigos naturais (grutas, cavernas e ocos de árvores) desde que se mantenha uma tem- peratura média entre 21 e $23^{\circ} \mathrm{C}$ e um mínimo de $45 \%$ de umidade (Flores-Crespo \& Arellano-Sota 1991).

Lord (1988) evidenciou que certas características de uma região determinam altas populações de $D$. rotundus. Segundo o autor, as áreas próximas aos principais rios geralmente favorecem a existência de um número maior de abrigos. Da mesma forma, Taddei et al. (1991), em estudo em escala estadual, relataram que os principais rios do estado de São Paulo foram as principais feições geográficas relacionadas à presença do morcego e ao desdobramento de uma epidemia de raiva em bovinos na década de 1980, mencionando que os municípios paulistas banhados pelas principais bacias hidrográficas foram os mais atingidos pela epidemia.

Os estudos de Taddei et al. (1991) estimularam os profissionais da Coordenadoria de Defesa Agropecuária (CDA), órgão responsável pelo controle da raiva dos bovinos no estado de São Paulo, a preconizar que as áreas próximas as principais bacias hidrográficas do estado deveriam ser consideradas como as mais importantes para o desenvolvimento de abrigos, e conseqüientemente de epidemias de raiva em bovinos (Peres et al. 2001). Assim, nas regiões de vigilância da enfermidade, vincularam as ações de controle às áreas próximas aos principais rios.

As decisões dos técnicos da CDA e de diversos estudos elaborados para verificar relações entre os morcegos vampiros, a raiva bovina e o ambiente não se beneficiaram de tecnologias de geoinformação que incluem Sistemas de Informação Geográfica (SIG), como os desenvolvidos por Lord (1988) e Taddei et al. (1991). A análise por meio de SIG possibilita integrar informações referentes aos fatores ambientais associados a uma enfermidade para o mapeamento de zonas propícias para a sua ocorrência, contribui na prevenção e controle dos agravos decorrentes da moléstia, no controle das populações de reser-

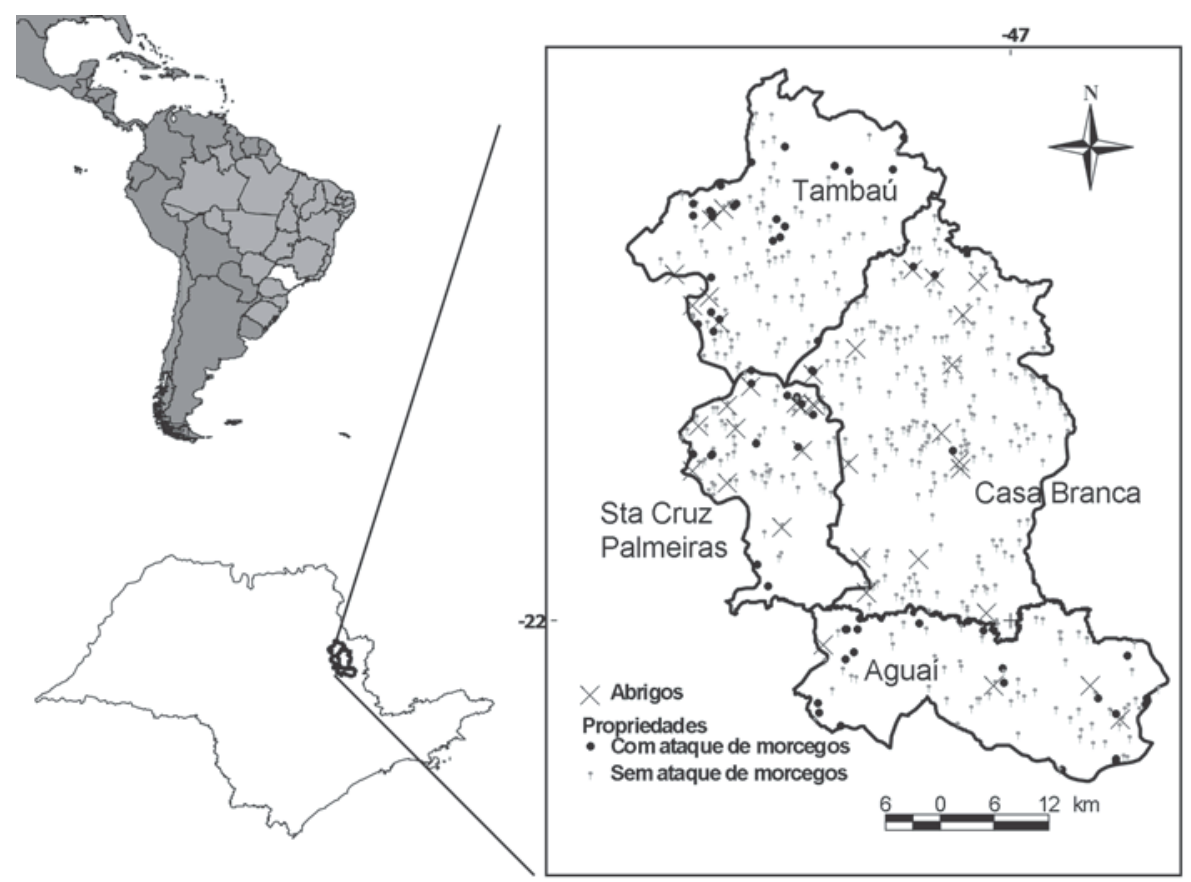

Fig.1. Área de estudo, localização por receptor de GPS da sede das propriedades com ataque de morcegos, propriedades sem ataque de morcegos e dos abrigos cadastrados. 
vatório e também, pode levantar novas hipóteses acerca da história natural de uma doença (Correia et al. 2004).

Um trabalho de controle da raiva dos herbívoros foi desenvolvido pela CDA com o georeferenciamento de propriedades e abrigos de morcegos vampiros na região de São João da Boa Vista além das áreas próximas aos principais rios, entre março e maio de 2003, denominado "mutirão de controle da raiva". Este trabalho possibilitou o desenvolvimento de um banco de dados geográfico dos principais eventos envolvidos ao ataque espoliativo de morcegos hematófagos em bovinos, e assim as informações obtidas foram integradas em um SIG para posterior análise.

Dessa maneira, objetivou-se verificar a hipótese de Taddei et al. (1991) que considera a relação dos abrigos de morcegos $D$. rotundus à proximidade dos principais rios e também, como objetivos específicos: (1) Gerar mapas de áreas propícias para o ataque espoliativo de morcegos hematófagos em bovinos; (2) Verificar se há concordância entre as áreas de ação de morcegos nos municípios de Aguaí, Casa Branca, Santa Cruz das Palmeiras e Tambaú (Fig.1), denominadas neste trabalho por "áreas de risco conhecidas", e as áreas de distância de até 3 km dos principais rios, denominadas neste trabalho por "áreas de risco estabelecidas".

\section{MATERIAL E MÉTODOS}

Um artigo anteriormente publicado (Gomes et al. 2005) foi desenvolvido com boa parte da metodologia utilizada neste trabalho. Para facilitar o seu entendimento e como ambos possuem materiais e métodos comuns, aqui, será enfatizada a descrição dos novos métodos utilizados unicamente para este estudo. Aqueles já descritos no trabalho anterior serão apenas sucintamente mencionados.

\section{Características ambientais dos municípios estudados}

As principais atividades agropecuárias dos municípios estudados incluem o cultivo de tubérculos (batata), citros e cana-de-açúcar. Esses municípios apresentam, de uma maneira geral, um aumento da atividade canavieira em detrimento da pecuária, com a diminuição gradativa do efetivo bovino (IBGE 2004). As pastagens encontram-se dispersas pelo território, com predominância no município de Tambaú e nas áreas consideradas impróprias para a lavoura.

A principal bacia hidrográfica da região é a do rio Pardo, no limite entre os municípios de Mococa e Tambaú com seu afluente, o rio Tambaú. Outros rios importantes são o Itupeva, que atravessa o município de Aguaí, e o rio Jaguari-Mirim, que faz o limite entre Aguaí e Casa Branca.

\section{Base de dados e programas utilizados}

Os dados utilizados para a análise tiveram origem, principalmente, na base de dados da Fundação Instituto Brasileiro de Geografia e Estatística (IBGE) e do "mutirão de controle da raiva" realizado pelos técnicos da CDA. O banco de dados geográfico para a construção do modelo foi formado pelos seguintes temas:

- Cadastro de abrigos de morcegos encontrados georreferenciados por receptor de GPS;

- Hidrografia (escala 1:100.000) dos rios de maior ordem da região (rios Pardo, Jaguari-Mirim, Tambaú e Itupeva);

- Cadastro de propriedades da região cujas sedes foram georreferenciadas por receptor de GPS e que possuíam o atributo número de bovinos mordidos;

- Malha Municipal (escala 1:100.000);

- Número de bovinos cadastrados por Município/IBGE no ano de 2003;

- Imagens de satélite LANDSAT/ETM - 219/75 (23.4.03) e 220/75 (30.4.03).
Os programas utilizados foram: SPRING para edição dos mapas e análises espaciais, Excel para edição do banco de dados e Epilnfo para análises estatísticas.

\section{Determinação das áreas de risco conhecidas}

As áreas de risco conhecidas foram geradas a partir da integração de mapas pela técnica AHP, Processo Analítico Hierárquico ou em inglês, Analytical Hierarchy Process (AHP) (Saaty 1992). Para tanto, foram utilizados três mapas previamente construídos: (1) mapa da distância aos abrigos dos morcegos, (2) mapa da distribuição dos bovinos atacados, e (3) mapa da distribuição de bovinos.

\section{Construção dos mapas e técnica AHP}

1) O Mapa da distância aos abrigos foi gerado a partir de "buffers" de raio de $3 \mathrm{~km}$ dos abrigos cadastrados. Essa distância foi baseada no raio de

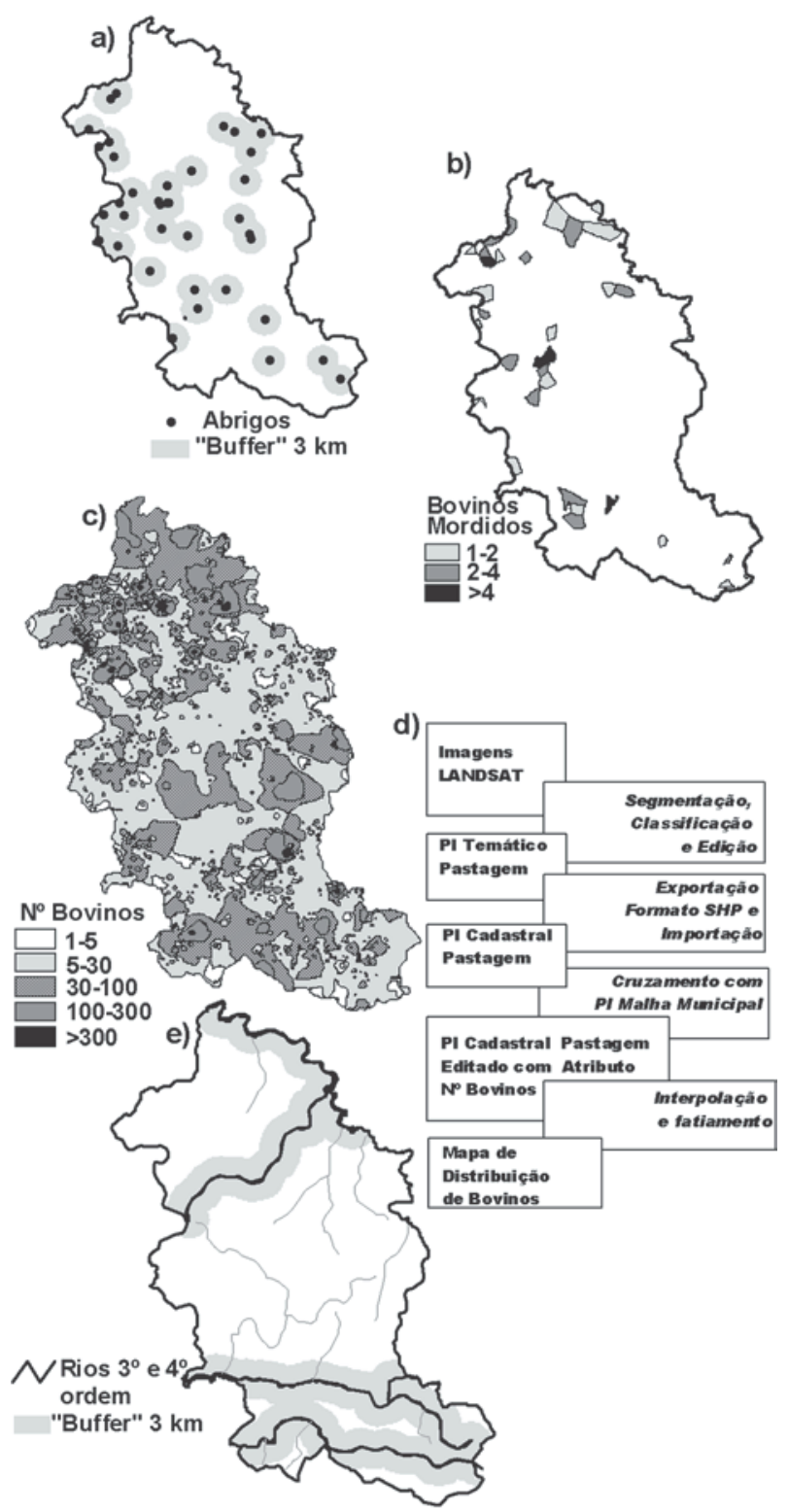

Fig.2. a) Mapa de distância dos abrigos, b) mapa de distribuição de bovinos mordidos, c) mapa de distribuição de bovinos, d) fluxograma para obtenção do mapa de distribuição de bovinos [PI= Plano de Informação, "layer"], e) mapa de distância dos rios de $3^{\underline{a}}$ e $4^{\mathrm{a}}$ ordem. 
Quadro 1. Resultado dos valores dos pesos atribuídos aos fatores considerados pela análise AHP

\begin{tabular}{lcc}
\hline \multicolumn{1}{c}{ Mapa } & Fator considerado & $\begin{array}{c}\text { Pesos } \\
\text { atribuídos }\end{array}$ \\
\hline Distribuição de bovinos mordidos & № de bovinos agredidos & 0,595 \\
Distância aos abrigos & Distância aos abrigos & 0,340 \\
Distribuição de bovinos & № de bovinos & 0,065
\end{tabular}

Quadro 2. Ponderação estipulada para cada fator considerado

\begin{tabular}{lcc}
\hline \multicolumn{1}{c}{ Fator considerado } & Valor & Ponderação estipulada \\
\hline Número de bovinos agredidos & $0-1$ & 0 \\
& $1-2$ & 0,3 \\
& $2-4$ & 0,7 \\
Distância aos abrigos & $>4$ & 1 \\
Número de Bovinos & $0-3 \mathrm{~km}$ & 0,8 \\
& $0-1$ & 0 \\
& $1-5$ & 0,08 \\
& $5-30$ & 0,3 \\
& $30-100$ & 0,6 \\
& $100-300$ & 0,8 \\
& $>300$ & 1
\end{tabular}

vôo noturno médio da espécie Desmodus rotundus (Wilkinson 1988, FloresCrespo \& Arellano-Sota 1991, Trajano 1996) (Fig.2a).

2) O Mapa da distribuição de bovinos atacados foi construído a partir da interpolação do número de bovinos mordidos (variaram entre 0 e 7) das propriedades atacadas por $D$. rotundus. Após a experimentação de alguns interpoladores, o escolhido foi o vizinho mais próximo (Burrough \& MacDonell 1998), pois ele possibilitava a maximização da área de bovinos mordidos, produzindo os melhores resultados (Fig.2b).

3) O Mapa da distribuição de bovinos (Fig.2c) foi elaborado a partir de interpolação e primeiramente, da agregação do número de bovinos dos municípios aos polígonos de áreas de pastagem (1680 polígonos) que foram obtidas a partir da classificação das imagens de satélites. Assim, o número de bovinos pôde estar desagregado dos polígonos municipais e associado aos de pastagem (ambiente natural dos bovinos), possibilitando uma análise mais próxima da realidade e em escala mais fina.

De fato, uma série de etapas foi seguida para se obter efetivamente o mapa de distribuição de bovinos. Estas etapas estão apresentadas resumidamente na Fig. $2 \mathrm{~d}$ e foram detalhadamente descritas por Gomes et al. (2005).

A técnica AHP é uma técnica de escolha baseada na lógica da comparação pareada. Nesse procedimento, os diferentes fatores considerados para formar um mapa de classificação de risco são comparados dois-a-dois de acordo com uma escala qualitativa de pesos atribuída pelo pesquisador.

Após a determinação da escala qualitativa, a técnica AHP calcula um peso de importância relativa ao relacionamento entre esses fatores. Os valores dessa relação são utilizados como dado de entrada em uma matriz de comparação pareada, onde é calculado um valor de razão de consistência do julgamento efetuado (com 0 indicando a completa consistência do processo de julgamento). Posteriormente, os dados são transformados para o espaço contínuo de referência $[0,1]$ e processados por combinação numérica de acordo com uma ponderação estipulada, e por fim, obtêm-se os valores de risco sob forma de uma grade numérica.

Neste trabalho, considerou-se maior importância qualitativa para o fator "número de bovinos agredidos", em seguida "a distância aos abrigos", e por último, o fator "número de bovinos". Os pesos de importância relativa atribuídos pela técnica AHP estão demonstrados no Quadro 1, a razão de consistência calculada foi de 0,016 e a ponderação estipulada aplicada pelo pesquisador está demonstrada no Quadro 2.

Assim, dos três mapas utilizados na técnica AHP para gerar o mapa de risco conhecido (Fig.3a), após diferenciação em classes, obteve-se, as áreas discriminadas de baixo, médio e alto risco para o ataque espoliativo de morcegos em bovinos.

\section{Determinação das áreas de risco estabelecidas}

As áreas de risco estabelecidas foram geradas a partir da integração de mapas por uma inferência Booleana (Bonham-Carter 1994). Para tanto, foram utilizados dois mapas previamente construídos: (1) mapa da distribuição do número de bovinos e (2) mapa de distâncias aos principais rios.

\section{Construção dos mapas e inferência Booleana}

1) $\mathrm{O}$ mapa da distribuição do número de bovinos foi o mesmo do item 3 - Mapa da distribuição de bovinos da "Geração dos mapas e técnica AHP" e sua construção já foi demonstrada;

2) O mapa de distância aos principais rios foi obtido a partir da elaboração de "buffers" das localizações dos rios de $3^{\mathrm{a}}$ a $4^{\mathrm{a}}$ ordem (Fig.2e). Baseada nos critérios utilizados pelos técnicos da CDA e raio de vôo de D. rotundus (Wilkinson 1988, Flores-Crespo \& ArellanoSota 1991, Trajano 1996) a distância única para a construção dos "buffers" foi de $3 \mathrm{~km}$.
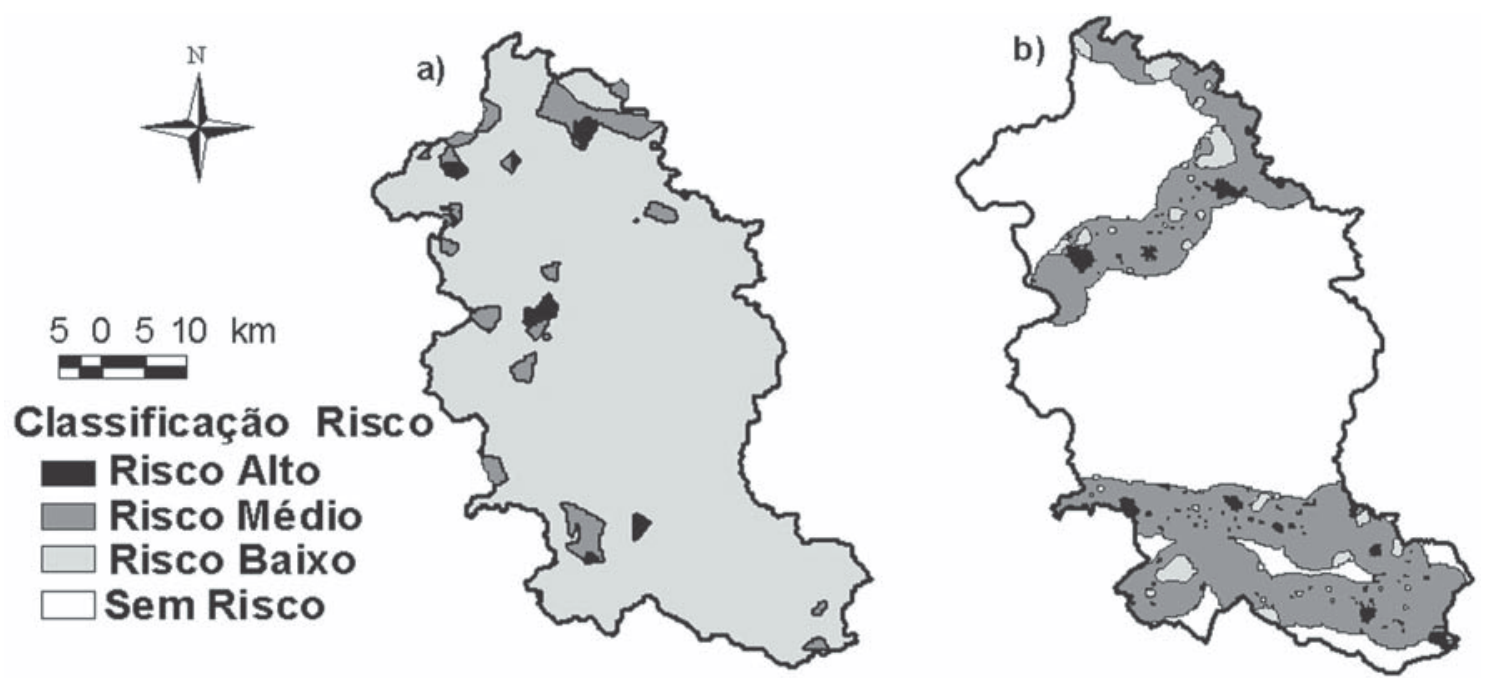

Fig.3. a) Mapa de risco conhecido, b) mapa de risco estabelecido. 
A inferência Booleana foi escolhida para gerar as áreas de risco estabelecidas em virtude dela possibilitar o uso da forma lógica binária que melhor representa o modo como os funcionários da CDA vinham trabalhando (atuação até uma distância fixa de $3 \mathrm{~km}$ dos principais rios "E" considerando maior risco para propriedades com maior número de bovinos). Assim, dos dois mapas utilizados para gerar o mapa de risco estabelecido (Fig.3b) obteve-se, após diferenciação em classes, as áreas discriminadas sem risco, baixo, médio e alto risco para o ataque espoliativo de morcegos em bovinos.

\section{Análises}

Para o cumprimento do primeiro objetivo específico foram gerados os mapas de risco conhecido e estabelecido. Para o cumprimento do segundo objetivo, primeiramente, foi calculado e comparado o total de áreas de risco (em hectares) de cada classe em cada mapa; como uma segunda forma de confrontar os dois mapas, desenvolveu-se uma matriz de confusão.

Para isso, os dois mapas de risco foram transformados para o formato matricial, constituindo-se por várias células "pixels" com resolução $500 \times 500 \mathrm{~m}$. Foi avaliado se as células de ambos eram plenamente coincidentes no espaço (possuíam as mesmas coordenadas) e, verificado se cada uma ficou identificada com uma das classes de risco. A referência da matriz de confusão foi o mapa de risco conhecido e o analisado o mapa de risco estabelecido (Quadro 4). Por coerência de análise e para que fosse possível a comparação dos dois mapas, uniu-se a classe de risco baixo e sem risco do mapa de risco estabelecido, ficando as duas denominadas simplesmente de risco baixo. A aleatoriedade foi estabelecida com a escolha de 100 coordenadas (posições) de células de cada classe do mapa de risco conhecido (referência) a partir da função "números aleatórios" do pacote estatístico do programa Excel. Calcularam-se, em seguida, o coeficiente Kappa e sua significância avaliada pelo valor do $Z$ estatístico, concordância de cada classe de risco, concordância global, os erros de omissão e inclusão (Vieira 2003).

\section{RESULTADOS}

Os mapas de risco conhecido e estabelecido com as respectivas áreas de risco estão apresentados na Fig.3. Os valores das dimensões das áreas classificadas como risco alto, médio e baixo, assim como, o número total de células de cada classe de risco de cada mapa constam no Quadro 3. Nele, verifica-se que o mapa de risco estabelecido apresentou maiores áreas e número de células para a classe de risco alto e médio que o mapa de risco conhecido, e o contrário para a classe de risco baixo.

O coeficiente Kappa calculado foi de $16 \%$, revelando uma concordância classificada como ruim (Pax-Lenney \& Woodcock 1997). A concordância global entre os dois mapas foi de $44 \%$. A concordância, erros de omissão e inclusão de cada classe de risco estão demonstrados no Quadro 4.

$O$ valor obtido do $Z$ estatístico foi de 5.10, ou seja, o resultado da classificação é estatisticamente superior ao obtido por uma classificação aleatória em um nível de confiança de 95\%, conclui-se que o resultado do coeficiente Kappa é estatisticamente significativo.

\section{DISCUSSÃO}

Um primeiro aspecto a ser considerado está relacionado com a classificação de imagens de satélite em áreas de pastagens para desagregar os dados da população de bovinos. Embora a classificação não tenha sido submetida a um teste de acurácia, ela se mostrou bastante útil e deve ser objeto de estudos futuros. Diferente dos estudos de Taddei et al. (1991), teve-se a iniciativa de agregar o número de bovinos às áreas de pastagem em virtude dos processos ambientais e sociais que promovem ou restringem situações de risco à saúde estarem associados a eventos dispersos pelo espaço, e não restritos aos limites administrativos criados pelo poderes públicos (Barcellos et al. 2003). Este trabalho se propôs a desafiar essa situação, buscando mudar a agregação do valor do número de bovinos oriundo do censo agropecuário do IBGE (e reavaliações anuais) que está associado à área (polígono) de cada município e associá-lo às áreas de pastagens, onde naturalmente os bovinos estão situados (bovinos confinados foram desconsiderados) melhorando a representação computacional do modelo.

Discutindo especificamente os resultados obtidos, verifica-se inicialmente por uma análise das Fig.3a e 3b, que as áreas de risco alto e médio dos mapas de risco estabelecido e conhecido pouco concordaram em localização e dimensão. A partir de uma visão analítica dos resultados apresentados nos Quadros 3 e 4, observa-se uma discrepância considerável entre os mapas de risco conhecido e estabelecido. $\mathrm{O}$ valor insatisfatório do coeficiente de Kappa, a baixa concordância observada nas principais classes de risco (risco alto e risco médio) a baixa concordância global e os valores dos erros de inclusão e omissão demonstraram que para a região e época analisadas, caso fossem realizados trabalhos de controle da população de morcegos Desmodus rotundus de acordo com a distância aos principais rios, os resultados poderiam ser pouco efetivos.

Considerando as duas principais classes de risco, o valor médio obtido do erro de inclusão de aproximadamente 50\% mostra que as equipes de controle da raiva da CDA iriam trabalhar erroneamente a metade das principais áreas consideradas de risco (36.671 ha) caso seguissem o mapa de risco

Quadro 3. Valores das áreas em hectares e número de células segundo a classificação de risco e os mapas analisados

\begin{tabular}{|c|c|c|c|c|}
\hline \multirow[t]{3}{*}{ Risco } & \multicolumn{4}{|c|}{ Mapas } \\
\hline & \multicolumn{2}{|c|}{ Conhecido } & \multicolumn{2}{|c|}{ Estabelecido } \\
\hline & Hectares & Células & Hectares & Células \\
\hline Alto & $2.007,13$ & 101 & $3.244,12$ & 152 \\
\hline Médio & $8.310,21$ & 412 & $70.098,88$ & 2.803 \\
\hline Baixo & $209.700,27$ & 8.286 & $146.674,60$ & 5.844 \\
\hline Total & $220.017,61$ & 8.799 & $220.017,61$ & 8.799 \\
\hline
\end{tabular}

Quadro 4. Matriz de confusão do número de células de cada classe entre o mapa de risco conhecido (referência) e o mapa de risco estabelecido (analisado)

\begin{tabular}{cccccccc}
\hline Conhecido & Alto & Médio & Baixo & Total & Concordância & Inclusão & Omissão \\
\hline Alto & 1 & 1 & 4 & 6 & $1 \%$ & $83,3 \%$ & $99 \%$ \\
Médio & 2 & 42 & 9 & 53 & $42 \%$ & $20,7 \%$ & $58 \%$ \\
Baixo & 97 & 57 & 89 & 243 & $89 \%$ & $63,3 \%$ & $13 \%$ \\
Total & 100 & 100 & 100 & 300 & & &
\end{tabular}


estabelecido. Ainda, em virtude do valor médio de erro de omissão calculado, observa-se que 78\% (8.047 ha) das áreas que deveriam ser trabalhadas segundo as áreas de risco alto e médio conhecidas, não teriam qualquer ação de controle dos técnicos da CDA. Por fim, avaliando a concordância somada das duas principais classes, verifica-se que dos 73.343 hectares segundo o mapa de risco estabelecido, apenas 31.537 hectares $(43 \%)$ seriam corretamente trabalhados, ou seja, menos da metade da área que deveria sofrer intervenção para o controle do ataque espoliativo de morcegos em bovinos.

Assim sendo, caso fosse realizado o controle de população de Desmodus rotundus conforme o mapa de risco estabelecido e logo em seguida houvesse a presença do vírus rábico entre os morcegos na região, provavelmente não se obteria o efeito desejado e o grassamento de uma epidemia em bovinos possivelmente ocorreria, mesmo realizando trabalhos de controle das populações próximas aos principais rios.

Nos estudos realizados por Fornes et al. (1974), no norte árido da Argentina, relataram-se que os principais abrigos ocupados pelos morcegos hematófagos eram constituídos principalmente por cisternas de fazendas, e que uma epidemia migratória de raiva em bovinos seguia o rumo das localizações desses abrigos. Este estudo sugere que $D$. rotundus pode sobreviver e sustentar uma epidemia mesmo vivendo em abrigos artificiais e distantes dos rios.

Os trabalhos de Gonçalves et al. (1996) constataram que $70 \%$ dos abrigos de morcegos hematófagos cadastrados pela CDA são artificiais, independentemente da sua localização. Germano et al. (1992) relataram que o $D$. rotundus possui uma grande adaptabilidade às ações do homem no ambiente. Assim, pode-se acreditar que com a ação humana na paisagem da região estudada, os $D$. rotundus acabariam não vivendo necessariamente próximos ao seu habitat preferencial perto dos principais rios. Poderiam, então se adaptar as outras áreas que originalmente não eram habitadas. Conseqüentemente não produziriam uma dispersão espacial de uma epidemia em bovinos próxima aos principais rios da região.

Taddei et al. (1991) relataram que nas estações mais frias e secas ocorrem deslocamentos de colônias de morcegos para regiões próximas aos principais rios paulistas Os dados do presente estudo não concordam com os dos autores citados, pois foram coletados no início da estação seca e portanto, poderia ter sido registrado um maior número de abrigos às margens dos principais rios. Ressalta-se que, na região estudada, além de abundância de abrigos artificiais, existe uma rede de rios permanentes de primeira e segunda ordem que podem produzir um ambiente adequado para $D$. rotundus, evitando a necessidade de grandes deslocamentos dos morcegos entre abrigos.

Uma característica importante das epidemias de raiva em bovinos é seu aspecto migratório (Fones et al. 1974). Os dados utilizados para a confecção deste trabalho não permitem uma análise muito ampla de uma dinâmica de difusão espacial de uma epidemia de raiva em virtude de terem sido coletados em apenas uma época, e assim, não se pode afirmar que uma possível epidemia ocorreria entre as áreas de risco conhecidas e não seguindo o rumo das áreas de risco determinadas.
Por outro lado, considerando-se que a conformação espacial dos abrigos dos morcegos se mantenha, o aspecto da epidemia poderia tomar caminhos difusos e não seguindo o curso dos principais rios, conforme a teoria de Taddei et al. (1991). Essas evidências concordam com o relato dos funcionários da CDA sobre o desenho da epidemia que se desenvolveu nos últimos cinco anos na região da Serra da Mantiqueira.

Ainda discutindo sobre epidemias migratórias, modelos matemáticos ou de processos procuram mimetizar a cadeia epidemiológica das enfermidades, simulando a complexa relação desde a fonte de infecção até os susceptíveis (Massad et al. 2004). Esses modelos são empregados, entre outras razões, para calcular a velocidade de propagação da infecção entre comunicantes, e da difusão espacial da enfermidade (geralmente objetivando criar um cordão de isolamento ou calcular o tempo disponível para as ações necessárias). Dessa forma, Anderson et al. (1981) e Murray et al. (1986) estudando raiva em raposas na Europa; e Childs et al. (2000) e Russel et al. (2005) pesquisando raiva em racoons nos Estados Unidos, elaboraram esses modelos para investigar a difusão espacial da raiva nas espécies estudadas.

O problema apontado no presente trabalho sugere a criação desses tipos de modelos, em princípio, para responder a questão aqui levantada, um modelo descritivo conforme construído foi suficiente. Para a mesma região, os dados levantados servirão de apoio para um modelo de difusão espacial que deverá ser gerado com dados de casos de raiva para o estado de São Paulo.

Um último aspecto que deve ser considerado diz respeito a diferença na escala de análise entre o presente trabalho e o de Taddei et al. (1991). Esses autores podem ter chegado a conclusão da relação dos municípios com maior número de focos de raiva em bovinos com as principais bacias hidrográficas do estado de São Paulo em função da escala pequena em que foram efetuadas suas análises. Neste estudo, a escala de trabalho utilizada foi próxima de 1:100.000. Portanto, pôde-se identificar detalhes mais específicos de uma determinada região, porém em uma área menor de estudo. 0 georreferenciamento de fazendas com animais agredidos e abrigos de morcegos, assim como a classificação de polígonos de pastagem permitiram investigações mais detalhadas. Neste sentido, conforme descrito em Cao \& Lam (1997) evidencia-se que a análise de eventos semelhantes em escalas diferentes pode produzir resultados distintos e a sua utilização prática deve ser interpretada e utilizada com cautela.

\section{CONCLUSÕES}

A hipótese de Taddei et al. (1991) que relaciona abrigos de morcego hematófago e epidemias de raiva em bovinos com os municípios banhados pelas principais bacias hidrográficas paulistas necessita maiores investigações. $O$ presente trabalho, assim como observações de campo, sugere que áreas próximas aos principais rios não necessariamente devem ser consideradas de risco para ataque de morcegos e que as estratégias de controle da raiva estabelecidas pelos técnicos da CDA necessitam ser reavaliadas. Os fatores que levam os morcegos hematófagos a formarem colônias em determinadas re-giões ainda não estão 
claros, e devem ser alvo de estudos mais específicos que preferencialmente integrem os SIG e escala de análise.

Agradecimentos.- A todos os funcionários da CDA envolvidos no controle da raiva dos herbívoros no estado de São Paulo, principalmente, ao Diretor da Regional de São João da Boa Vista, Pedro Valim, e aos funcionários Paulo e Arcanjo. Agradecemos, ainda, ao Dr. Wilson Uieda pela revisão deste trabalho.

\section{REFERÊNCIAS}

Acha P.N. \& Málaga-Alba M. 1988. Economic losses due to Desmodus rotundus, p.208-213. In: Greenhall A.M. \& Schmidt U. (ed.), Natural History of Vampire Bats. CRC Press, Boca Raton.

Almeida M.F., Aguiar E.C.E., Martorelli L.F.A. \& Silva M.M.S. 1994. Diagnóstico laboratorial de raiva em quirópteros realizado em área metropolitana na região sudeste do Brasil. Revta Saúde Pública 28:341-344.

Anderson R.M., Jackson H.C., May R.M. \& Smith A.M. 1981. Population dynamics of fox rabies in Europe. Nature 289:765-771.

Barcellos C., Lammerhirt C.B., Almeida M.A.B. \& Santos E. 2003. Distribuição espacial da leptospirose no Rio Grande do Sul, Brasil: recuperando a ecologia dos estudos ecológicos. Cad. Saúde Pública 19(5):234-242.

Bonham-Carter G.F. 1994. Geographic Information Systems for Geoscientists. Pergamon/Elsevier Science, Terrytom. 185p.

Burrough P.A. \& MacDonell R.A. 1998. Principles of Geographic Information Systems. Oxford University, Oxford. 333p.

Brass D.A. 1994. Rabies in Bats: natural history and public health implications. Livia Press, Connecticut. 352p.

Cao C. \& Lam N. 1997. Understanding the scale and resolution effects in remote sensing and GIS, p.57-72. In: Quattrochi D.A \& Goodchild M.F. (ed.), Scale in Remote Sensing and GIS. CRC Lewis, Boca Raton.

Childs J.E., Curns A.T., Dey M.E., Real L.A., Feinstein L., BjØrnstad O.N. \& Krebs J.W. 2000. Predicting the local dynamics of epizootic rabies among raccoons in the United States. PNAS 97(25):13666-13667.

Correia V.R.M., Carvalho M.S., Sabroza P.C. \& Vasconcelos C.H. 2004. Remote sensing as a tool to survey endemic diseases in Brazil. Cad. Saúde Pública 20(4):891-904

Delpietro H.A. \& Nader A.J. 1989. Rabies of herbivores transmitted by vampire bats in north-eastern Argentina. Rev. Sci. Off. Int. Epiz. 8(1):189-198.

Fornes A., Lord D.L., Kuns M.L., Larghi O.P., Fuenzalida E. \& Lazara L. 1974. Control of bovine rabies through vampire bat control. J. Wildlife Dis. 10:310316.

Flores-Crespo R. \& Areallano-Sota C. 1991. Biology and control of vampire bat, p.462-474. In: Baer G.M. (ed.), The Natural History of Rabies. CRC Press, Boca Raton.
Gomes M.N., Monteiro A.M.V., Nogueira-Filho V.S. \& Gonçalves C.A. 2005. O uso do Sensoriamento Remoto e de Sistemas de Informação Geográfica na análise de áreas de risco ao ataque de morcegos hematófagos em bovinos de quatro municípios da região de São João da Boa Vista, estado de São Paulo. Anais $12^{\circ}$ Simp. Bras. Sensoriamento remoto. SBSR, p.2733-2740. http://marte.dpi.inpe.br/col/ltid.inpe.br/sbsr/2004/11.19.07.35/doc/2733.pdf

Germano P.M.L., Germano M.I.S., Miguel O. \& Lagos C.B.T. 1992. O papel dos morcegos hematófagos na cadeia de transmissão da raiva silvestre. Comun. Científ. Fac. Med. Vet. Zootec. USP. 16(1):21-25.

Gonçalves C.A. 1996. Controle de populações de morcegos hematófagos no estado de São Paulo. Bolm Inst. Pasteur 2:45-49.

IBGE 2004. Fundação Instituto Brasileiro de Geografia e Estatística. < http:// www.sidra. ibge.gov.br> (acessado em 20/03/2004).

Lord R.D. 1988. Control of vampire bats, p.215-226. In: Greenhall A.M. \& Schmidt U. (ed.), Natural History of Vampire Bats. CRC Press, Florida.

Massad E., Menezes R.X., Silveira P.S.P. \& Ortega N.R.S. 2004. Métodos Quantitativos em Medicina. Manole, São Paulo. 561p.

Murray J.D., Stanley E.A. \& Brown D.L. 1986. On the spatial spread of rabies among foxes. Proc Roy. Soc. London, Series B 229:111-150.

Pax-Lenney M. \& Woodcock C.E. 1997. The effect of spatial resolution on the ability to monitor the status of agricultural lands. Remote Sensing of Environment 61(2):210-228.

Peres N.F., Carrieri M.L., Carniele-Jr P., Lazarini S.R.F., Diniz O. \& Silva A.S. 2001. Dinâmica da difusão da raiva dos herbívoros no estado de São Paulo. Anais Sem. Int. Morcegos como Transmissores da Raiva. Instituto Pasteur, São Paulo, p.29-30.

Saaty T.L. 1992. Multicriteria Decision Making: the analytical hierarchical process. RWS, Pittsburg. 125p.

Taddei A.V., Gonçalves C.A., Pedro W.A., Tadei W.J., Kotait I. \& Arieta C. 1991. Distribuição do morcego vampiro Desmodus rotundus no Estado de São Paulo e a raiva dos animais domésticos. Coordenadoria de Assistência Técnica Integral, Campinas. 107p.

Trajano E. 1996. Movements of cave bats in southeastern Brazil, with emphasis on the population ecology of the common vampire bat, Desmodus rotundus. Biotropica 28(1):121-129.

Russell C.A., Smith D.L., Childs J.E. \& Real L.A. 2005. Predictive Spatial Dynamics and Strategic Planning for Raccoon Rabies Emergence in Ohio. PLoS Biology 3(3):382-388.

Uieda W. 1987. Morcegos hematófagos e a raiva dos herbívoros no Brasil. Anais Sem. Ciênc. Faculdades Integradas de Uberaba, FIUBE. Uberaba, p.1329.

Vieira C.A.O. 2000. Accuracy of Remotely Sensing Classification of Agricultural Crops: a comparative study. Tese de Doutorado, University of Nottingham, Nottingham. 327p. 